\title{
PRÁCTICAS PEDAGÓGICAS INTERCULTURALES Y FORMACIÓN DE COMPETENCIAS CIUDADANAS EN LA EDUCACIÓN RURAL
}

\section{INTERCULTURAL PEDAGOGICAL PRACTICES AND TRAINING OF CITIZEN SKILLS IN RURAL EDUCATION}

\author{
María Elisa Álvarez Ossa ${ }^{1}$ \\ Fernando José Henao Franco ${ }^{2}$ \\ Juan Pablo García Giraldo ${ }^{3}$ \\ Albeiro Dávila Grisalez ${ }^{4}$ \\ Yeraldin Giraldo Isaza ${ }^{5}$ \\ Juliana Andrea Juspian Marín ${ }^{6}$
}

UCEVA, Universidad Central del Valle

1 Doctora en Ciencias de la Educación RUDECOLOMBIA, Magister en Educación de la Universidad Javeriana - Cali, docente de la Institución Universitaria Unidad Central del Valle del Cauca UCEVA, Líder Grupo de Investigación Educación y Currículo

mealvarez@uceva.edu.co.

ORCID ID. https://orcid.org/0000-0002-4140-7793

$2 \quad$ Magister en historia de la Universidad del Valle, Magister en Educación de la Universidad

Javeriana - Cali, estudiante de doctorado en Filosofía de la Universidad del Valle, docente hora catedra de la Institución Universitaria Unidad Central del Valle del Cauca UCEVA. Integrante Grupo de Investigación Educación y Currículo

fjhenao@uceva.edu.co.

ORCID ID. https://orcid.org/0000-0002-5799-5753

3 Magister en Estudios de Paz y Conflicto de la Universidad de Queensland, Australia, docente hora catedra de la Institución Universitaria Unidad Central del Valle del Cauca UCEVA. Integrante Grupo de Investigación Derechos Humanos y Víctimas en escenario de posconflicto.

correo electrónico: jpgarcia@uceva.edu.co

ORCID ID.

https://orcid.org/0000-0002-5897-0646

$4 \quad$ Magister en intervención integral en el deportista de la Universidad Autónoma de Manizales UAN, docente de la Institución Universitaria Unidad Central del Valle del Cauca UCEVA, integrante Grupo de Investigación Educación y Currículo. adavila@uceva.edu.co

ORCID ID: https://orcid.org/0000-0002-2508-7742

$5 \quad$ Licenciada en Educación Básica con Énfasis en Educación Física, Recreación y Deporte de la Institución Universitaria Unidad Central del Valle del Cauca UCEVA, integrante del Semillero de Investigación Prácticas Pedagógicas y Evaluativas del Grupo de Investigación Educación y Currículo, estudiante de la Maestría en Pedagogía de la UCEVA.

yeraldin.giraldo01@uceva.edu.co https://orcid.org/0000-0003-3529-6377,

$6 \quad$ Licenciada en Educación Básica con Énfasis Educación Física, Recreación y Deporte de la Institución Universitaria Unidad Central del Valle del Cauca UCEVA, integrante del Semillero de Investigación Prácticas Pedagógicas y Evaluativas del Grupo de Investigación Educación y Currículo.

Julianajuspian.1996@gmail.com https://orcid.org/0000-0001-9649-9819 


\section{RESUMEN}

El presente artículo se deriva de los resultados de una investigación ${ }^{7}$ que tuvo por objeto de estudio describir las prácticas pedagógicas interculturales y el desarrollo de competencias ciudadanas en instituciones educativas rurales de dos municipios del Centro del Valle del Cauca, Colombia. Se desarrolló bajo un enfoque mixto, con diseño no experimental de corte transversal de alcance descriptivo, mediante la aplicación de encuestas, entrevistas y el análisis documental sobre educación rural. Los resultados obtenidos permitieron evidenciar que los promedios en los resultados de la prueba Saber en competencias ciudadanas de los grados quinto de la zona rural son mayores que los obtenidos por los niños de la zona urbana y bajan cuando llegan al grado noveno y once. Se identificaron varios factores del contexto educativo rural, los más relevantes son el deterioro del tejido sociocultural y el proceso migratorio como consecuencia del conflicto armado y el narcotráfico que ha vivido el país; otro factor es la aplicación de un modelo educativo homogeneizante que genera pérdida de identidad con la cultura rural. Además, se concluye que la formación de competencias ciudadanas no es homogénea en los municipios objeto de estudio, pero en todas ellas se encontró que es fundamental formar en y para el diálogo edificando procesos culturales con identidad, alteridad y resiliencia.

PALABRAS CLAVE: competencias ciudadanas, practicas pedagógicas interculturales, educación rural, identidad, alteridad y resiliencia.

\section{ABSTRACT}

This article is the result of a research that its objective was to describe the pedagogical intercultural practices and the development of citizenship competences in rural educative

$7 \quad$ Prácticas pedagógicas interculturales que aportan a la formación de competencias ciudadanas y a la construcción de cultura política en instituciones educativas rurales de dos municipios del Centro del Valle del Cauca (Guadalajara de Buga y Tuluá). institutions of two cities in the center of Valle del Cauca in Colombia. It was developed through a mixed approach, with non-experimental design transversal and descriptive, by application of survey, interview and the documental analyzes about rural education. The obtained results allowed to make evidence that the average in the results of Prueba -saber in citizenship competence of the fifth grade in the rural area are higher than the ones obtained by the children in the urban area and they low when the students are in ninth and eleventh grade. It was identified several factors in the rural educative context, the most outstanding in the armed conflict and drug trafficking that has lived in the country; another factor is the application of an educative homogenizing model that generates lost of identity with a rural culture. Beside it is concluded that the education in the citizenship competences is not homogeneous in the cities focused in, but in all of them were found that is fundamental to form in and for the dialogue building cultural processes with identity, otherness and resilience.

KEYWORDS: citizen competences, pedagogical practices, interculturality.

\section{INTRODUCCIÓN}

La reflexión sobre la formación en competencias ciudadanas y la caracterización cultural de la población que habita el espacio rural, requiere de la lectura del contexto histórico bajo la lógica de las tensiones y dinámicas sociopolíticas, económicas y culturales; articulado al análisis de las prácticas pedagógicas desarrolladas en las instituciones educativas rurales, en el caso de esta investigación se realizó en los municipios de Guadalajara de Buga y de Tuluá, ubicados en el centro del departamento del Valle del Cauca. Se logró identificar la presencia de una población diversa en los grupos de estudiantes objeto de este estudio; también el predominio de un modelo educativo monocultural en la educación rural, que no es pertinente con la diversidad 
cultural de la población que habita el campo en Colombia a la luz de la Constitución de 1991. Se abordó la reflexión de una propuesta pedagógica intercultural que aporte a la formación de una ciudadanía activa que pueda reconocerse, dialogar y transformar con identidad, alteridad y resiliencia.

\section{METODOLOGÍA}

En cuanto al diseño metodológico utilizado en esta investigación, correspondió a un estudio mixto. Los resultados que se presentan corresponden al desarrollo de un diseño no experimental de corte transversal con alcance descriptivo, donde se definieron como variables de investigación las competencias ciudadanas y las prácticas pedagógicas interculturales. La investigación se realizó en instituciones educativas rurales de dos municipios del centro del Valle del Cauca, Colombia. Tuluá es un municipio con nueve (9) instituciones educativas rurales y Guadalajara de Buga tiene seis (6), para este estudio se seleccionó el $50 \%$ de ellas, que correspondió a cuatro (4) Instituciones de Tuluá y tres (3) Instituciones de Guadalajara de Buga. Las instituciones se codificaron de la siguiente manera: de Tuluá Institución Educativa L.M., Institución Educativa J.S., Institución Educativa S.R. e Institución Educativa AG. y de Guadalajara de Buga Institución Educativa N. S., Institución Educativa A.C., Institución Educativa MG.

El estudio se focalizó en primaria grado $5^{\circ}$ y en secundaria grado $9^{\circ}$ y $11^{\circ}$, y se le aplicó la encuesta al $30 \%$ de los estudiantes como instrumento de recolección de información cuantitativa y la entrevista se aplicó por conveniencia, es decir los estudiantes y docentes que accedieron brindar información: tres docentes y tres estudiantes de cada institución en total a 21 docentes y 21 estudiantes. Los criterios de inclusión fueron que las instituciones estuvieran ubicadas en la zona rural plana y de baja y media montaña, para la selección de los grados de escolaridad se tuvo en cuenta que correspondieran a los grados en que se aplica la prueba de estado Saber $5^{\circ}$, Saber $9^{\circ}$ y Saber $11^{\circ}$. El análisis documental se realizó revisando antecedentes de investigaciones sobre educación rural, estudios de interculturalidad y de formación en competencias ciudadanas.

\section{EL PROBLEMA Y SU CONTEXTO}

En Colombia los docentes y directivos dialogan mucho sobre la evaluación de la prueba Saber de los estudiantes de primaria y secundaria, se habla de la necesidad de generar una capacitación solo tomando como base la información de los resultados de la Institución educativa o compararlos con otras, pero sin contextualizar lo socio cultural. Sin embargo, poco se ha trabajado en aquellas situaciones de la vida escolar del estudiante de donde se desprenden sus experiencias, respuestas, preguntas y expectativas. Son múltiples los elementos que generan acercamientos $y$ diferencias en la convivencia en contexto rural, lo que se evidencia es una práctica de las competencias ciudadanas alejada de la cultural rural, es decir se ha ido perdiendo el sentido de la pluralidad, la diversidad, el diálogo y la identidad con las tradiciones de la población campesina. Además, en los procesos curriculares la tendencia es que a medida que se avanza en los grados escolares, cada vez más, se imparten contenidos culturales y académicos homogeneizantes mediante microcurrículos semejantes a los procesos educativos de las instituciones urbanas; lo que influye en las expectativas de los estudiantes, con proyectos de vida diferentes a la vida del campo. Además, falta que las políticas gubernamentales y sus estrategias de acción conlleven desarrollos socioeconómicos y tecnológicos que realmente impacten de manera esperanzadora los proyectos de vida de la población campesina sin alterar su cultura. 
Esta investigación realizo una compilación de resultados promedio en el componente de competencias sociales y ciudadanas de las instituciones rurales y urbanas en pruebas SABER $11^{\circ}$ de $2018-2$ y pruebas SABER grados $5^{\circ}$ y $9^{\circ}$ de 2015 , direccionado por el Ministerio de Educación Nacional (MEN) y el Instituto Colombiano para la Evaluación de la Educación Superior (ICFES interactivo).

Tabla 1 Resultados de competencias sociales y ciudadanas en pruebas saber $11^{\circ}$ de $2018-2$ y pruebas saber grados $5^{\circ}$ y $9^{\circ}$ de 2015 a nivel nacional departamental y municipal.

\begin{tabular}{lllllll}
\hline \multirow{2}{*}{ Entidad territorial } & \multicolumn{3}{c}{ Promedio } & \multicolumn{3}{c}{ Desviación } \\
\cline { 2 - 7 } & $5^{\circ}$ & $9^{\circ}$ & $11^{\circ}$ & $5^{\circ}$ & $9^{\circ}$ & $11^{\circ}$ \\
\hline Colombia & 298 & 292 & 49 & --- & --- & 12 \\
\hline Valle & 296 & 281 & 48 & --- & --- & 11 \\
\hline Guadalajara de Buga & 304 & 299 & 49 & -- & -- & 11 \\
\hline Tuluá & 316 & 304 & 50 & --- & -- & 11 \\
\hline
\end{tabular}

Fuente: tomado y adaptado de ICFES interactivo resultados-saber consultado marzo, 2019.

Como se puede observar en la tabla número 1 , el promedio de todas las instituciones a nivel municipal de la región central del Valle del Cauca al comparar los resultados con el nivel nacional y departamental se evidencia como superan el promedio, encabezando el municipio de Tuluá, siguiéndole Guadalajara de Buga.

Tabla 2 Comparación del promedio en los resultados de competencias sociales y ciudadanas en pruebas saber $11^{\circ}$ de 2018-2 y pruebas saber grados $5^{\circ}$ y $9^{\circ}$ de 2015 instituciones rurales y urbanas.

\begin{tabular}{|c|c|c|c|c|c|c|c|c|c|c|c|c|}
\hline \multirow{3}{*}{ Entidad territorial } & \multicolumn{6}{|c|}{ RURAL } & \multicolumn{6}{|c|}{ URBANO } \\
\hline & \multicolumn{3}{|c|}{ Promedio } & \multicolumn{3}{|c|}{ Desviación } & \multicolumn{2}{|c|}{ Promedio } & \multicolumn{4}{|c|}{ Desviación } \\
\hline & $5^{\circ}$ & $9^{\circ}$ & $11^{\circ}$ & $5^{\circ}$ & $9^{\circ}$ & $11^{\circ}$ & $5^{\circ}$ & $9^{\circ}$ & $11^{\circ}$ & $5^{\circ}$ & $9^{\circ}$ & $11^{\circ}$ \\
\hline Colombia & 277 & 262 & 45 & --- & --- & 10 & 291 & 286 & 49 & --- & --- & 11 \\
\hline Valle & 291 & 279 & 45 & --- & --- & 10 & 293 & 279 & 49 & --- & --- & 11 \\
\hline $\begin{array}{l}\text { Guadalajara de } \\
\text { Buga }\end{array}$ & 324 & 284 & 45 & --- & --- & 9 & 297 & 300 & 51 & --- & --- & 11 \\
\hline Tuluá & 303 & 279 & 46 & --- & --- & 10 & 301 & 296 & 49 & --- & --- & 11 \\
\hline
\end{tabular}

Fuente: tomado y adaptado de ICFES interactivo, Resultados-saber, consultado marzo, 2019. 
En la tabla 2 se describen los valores promedios de las competencias sociales y ciudadanas de las pruebas saber 11 de 2018-2 y las pruebas saber grados $5^{\circ}$ y $9^{\circ}$ de 2015 de instituciones rurales y urbanas, donde se observa cómo los promedios de los grados quinto de la zona rural son mayores que los promedios obtenidos por los niños de la zona urbana, pero a medida que avanzan en los niveles educativos los estudiantes de los grados noveno y once de la zona rural, al presentar las pruebas van disminuyendo en su promedio, al compararlos con los resultados de los estudiantes de la zona urbana.

En este orden de ideas, se analizó el porqué de esta tendencia de presentarse un decrecimiento en el desarrollo de las competencias ciudadanas, unido a la problemática que se vive en el contexto rural. Partiendo de varios estudios de la educación rural que muestran en diferentes formas las dinámicas de la población de la zona rural que son afectadas por el conflicto del desplazamiento de diferentes comunidades entre ellas campesinos, indígenas y afro. Como lo describe López (2006), la escuela se afecta con las problemáticas que se derivan de la migración campo - ciudad por el conflicto armado. Igualmente, otros estudios como el de Mejía (2011) plantean la incidencia de la cultura mafiosa por la incursión del narcotráfico comprando extensas y lujosas propiedades, cambiando las formas de producción de manera legal hacia lo ilegal por su rentabilidad, afectando los estilos de vida, las tradiciones y las formas de convivencia ciudadana de las comunidades rurales. También, inciden las desventajas del campo con relación a la ciudad referente a la garantía de derechos básicos como son salud, educación, vivienda y oportunidades laborales; en lo educativo por la suspensión parcial o definitiva de actividades escolares por orden público, situaciones que llevan al campesino a abandonar sus territorios y tradiciones culturales. Al respecto, Perfetti (2003) presenta un estudio sobre la situación educativa, social y económica de las zonas rurales del país y describe algunas experiencias significativas de educación en el campo.

Además, para abordar el análisis de la caracterización cultural de la población estudiantil rural de las instituciones objeto de este estudio, se inicia la reflexión partiendo de la caracterización poblacional identificada en el Censo Nacional de población y vivienda aplicado en el año 2018 (DANE, 2019), en el cual fue fundamental la autoidentificación o autorreconocimiento. La población autorreconocida como NARP (negros, afrodecesdientes, raizales y palenqueros fueron 4.671 habitantes, que corresponden al $(9,34 \%)$ de la población nacional, de los cuáles $3.533(66,7 \%)$ viven en las cabeceras municipales y $1.138(33,3 \%)$ en los centros poblados o área rural dispersa. Con respecto a la población indígena se autorreconocen 1.905.617 habitantes, representando el $(4,4 \%)$ de la población total del país, de los cuáles $400.180(21 \%)$ viven en centro poblado o área rural dispersa y $1.505 .437(79 \%)$ en la cabecera municipal.

Los procesos culturales en la zona rural se enriquecen y complejizan con la diversidad de la población que en ella habitan, conviven diferentes comunidades con sus propias formas de vida, (campesinos, indígenas, afros, los desplazados de otras regiones o los migrantes de otros países, los propietarios de extensas cantidades de tierra, los dueños de casas de campo como lugar de recreación, los comerciantes, entre otros). Esta variada composición poblacional por la llegada de diferentes grupos sociales y étnicos al campo, con intereses diferentes requieren propuestas de educación intercultural que aporte elementos a algo que pueden y deben tener en común los habitantes del campo mediante procesos de alteridad y resiliencia que hagan posible defender la tierra, rescatar la identidad cultural, 
garantizar la convivencia, sostener la seguridad alimentaria; mediante una relación de respeto y equilibrio con la naturaleza.

Teniendo en cuenta los planteamientos anteriores se realizó el proceso de reflexión e indagación desde el siguiente interrogante ¿Qué prácticas pedagógicas interculturales aportan a la formación de competencias ciudadanas en instituciones educativas rurales de dos municipios del centro del Valle del Cauca?

\section{ANTECEDENTES}

Se hizo un análisis a partir de la revisión sistemática en bases de datos científicas y en otras fuentes sobre las categorías centrales de este estudio, se utilizó como estrategia para la búsqueda e identificación de antecedentes las siguientes palabras clave: interculturalidad, práctica pedagógica, educación rural y competencias ciudadanas.

Con respecto a la interculturalidad y las prácticas pedagógicas interculturales:

El artículo titulado "Una reflexión crítica desde la educación intercultural hacia la pedagógica de la recreación" (Alomía, 2015) ha tenido como objetivo principal formular un marco de fundamentación epistemológica conducente a una pedagogía de la recreación a partir de su incidencia formativa en lo socioeducativo y cultural aportando al desarrollo individual, grupal y comunitario. Se presenta la perspectiva critico social como alternativa que aporta al desarrollo de las relaciones sociales interculturales con equidad y diálogo en las prácticas corporales, socio motrices y recreativas; porque la recreación desde la perspectiva intercultural es una herramienta didáctica importante en las interrelaciones humanas, es presentada como transformadora aportando a los procesos de formación integral.
El artículo titulado "Modelos y enfoques de educación intercultural" (Sánchez, 2011), tiene como objetivo exponer los modelos y enfoques interculturales que se han utilizado hasta la fecha y la incidencia de la aplicación de estos en los contextos educativos, también, se presenta una analogía de cómo se caracteriza el modelo multicultural y el intercultural. Explica la importancia de pasar del enfoque multicultural al enfoque intercultural donde en el primero, prevalece la tolerancia a la diferencia desde la racionalidad monológica que no reconoce al otro como interlocutor válido, en la expectativa de convivir en la diferencia sin agresión. En el segundo enfoque se enfatiza el reconocimiento entre las culturas, generando sociedades integradas que promueven encuentros dialógicos en medio de las diferencias culturales lo que potencia las identidades de cada comunidad étnica. Se presentan modelos interculturales que se han aplicado en contextos socioeducativos.

El artículo "La educación intercultural ante el desplazamiento forzado: ¿Posibilidad o quimera? (Albán, 2012), plantea que la educación en Colombia debe responder a la interculturalidad, la cual no debe ser la misma para todos, contemplando la diversidad, frustraciones, terrores y desarraigos, desde el reconocimiento de los derechos, por medio de una investigación cualitativa-descriptiva, el autor busca analizar la relación entre educación intercultural y los elementos que esta brinda al educando en situación de desplazamiento, quienes continuamente se ven afectaos por la discriminación y la estigmatización. Concluye que, la problemática del desplazamiento forzado en Colombia no es un fenómeno actual, ha acompañado las diferentes etapas de violencia que ha vivido la Nación. También expone que, aunque se han realizado experiencias pedagógicas direccionadas a la población desplazada sobre el respeto, la dignidad y el reconocimiento de sus costumbres, saberes culturales y ancestrales, es importante seguir 
trabajando por una Educación que responda a las necesidades de toda la población y no solo de grupos poblacionales social y económicamente privilegiados.

El de Pinzón (2010), “Anotaciones para una pedagogía intercultural en contextos urbanos" caracteriza los criterios de abordaje para una propuesta de educación intercultural en el ámbito de la escuela colombiana que supere el simple reconocimiento de la diversidad cultural, porque ve la necesidad de una educación intercultural de reconocimiento recíproco, diálogo y consenso en la toma de decisiones. Además, expone una caracterización cultural de la escuela colombiana en tres tipologías: la escuela de la parroquia, la del palenque y la de la maloca.

Guerrero (2014) plantea tres tendencias de los procesos educativos interculturales que terminan generando una reproducción del colonialismo epistémico, ontológico y cultural que se ha ido institucionalizando: 1) europea: la culturización del otro, 2) norteamericana: la mercantilización del otro y 3) latinoamericana: la emulación del otro.

El artículo "interculturalidad y política educativa en Colombia" (Guido, 2010) presenta tres posturas de la educación intercultural: 1) la relacional que plantea un intercambio cultural en términos de inferioridad y superioridad. 2) la funcional que incorpora grupos étnicos diferentes en una estructura social promoviendo diálogo y 3) la crítica que parte preguntas acerca de la discriminación racial y los intereses colonialistas de poder que requieren ser transformados para construir condiciones y posibilidades sociales diferentes. Además, presenta un análisis acerca de la de la interculturalidad en la política pública educativa en Colombia. La finalidad principal del artículo es de aportar, desde una postura crítica, alternativas que favorezcan el tratamiento de los procesos educativos y la diversidad cultural, teniendo en cuenta los cambios normativos y constitucionales que conllevan al reconocimiento del carácter multiétnico y pluricultural de la población y las luchas de los movimientos sociales, en documentos de política pública.

Con respecto a la práctica pedagógica se tuvieron en cuenta los siguientes antecedentes:

El artículo titulado "interculturalidad, practicas pedagógicas de maestros y maestras $y$ construcción del otro" (Riveros, 2010), corresponde a una investigación realizada en Bogotá que es una ciudad grande por ser el distrito capital de Colombia, se describen las prácticas de los docentes con un enfoque intercultural que se ha venido construyendo por parte de la secretaria de educación de Bogotá, además, expone una reflexión sobre la interculturalidad y su importancia en relación a la comunidad escolar con el fin de reconocer lo diverso y desarrollar una actitud de mutuo entendimiento de cultura que logra un enriquecimiento de identidad cultural en todas las diferentes comunidades y lograr desaparecer o disminuir las desigualdades sociales, por lo tanto, hay unas preguntas que suscitan de este avance y son: ¿en qué medida esos modelos de educación intercultural han contribuido a la transformación curricular? y ¿Cómo se han modificado las prácticas y vivencias de los docentes?

La investigación de Zuluaga, et al (2011) en su libro titulado "pedagogía y epistemología" presenta una variedad de artículos que recogen la sistematización teórica pedagogía elaboradas por el grupo de Historia de la Práctica Pedagógica, tiene como objeto de estudio reconocer la historia de la educación y la pedagogía en la sociedad colombiana y a su vez, reconocer su campo intelectual mediado por las tensiones entre las ciencias de la educación y la ciencia pedagógica en el orden universal y nacional. Lo anterior contribuye a los agentes y agencias educativas a comprender las practicas 
pedagógicas en la relación escuela, maestro y sociedad.

Igualmente, el Ministerio de Educación Nacional (2001) en el documento de lineamentos curriculares Cátedra estudios afrocolombianos presenta el trabajo en conjunto con las comunidades afrocolombianas, proponiendo: "la Identificación y conceptualización de prácticas de enseñanza y aprendizajes ancestrales de corte etnoeducativo para ampliar y enriquecer el debate pedagógico nacional sobre modelos educativos alternativos" (p.16). También, explica la necesidad de articular desde el proyecto educativo institucional el diseño curricular con enfoque etnoeducativo, la creación y uso de textos escolares de acuerdo a las realidades culturales de la comunidad y la importancia de formar en valores desde las cosmovisiones de los grupos étnicos.

Burgos \& Cifuentes (2015) en su artículo "La práctica pedagógica investigativa: entre saberes, quereres y poderes", analizan la práctica pedagógica como escenario donde confluye en el acto educativo el saber disciplinar, las estrategias didácticas, las relaciones de poder entre sus actores (principalmente los docentes, estudiantes y directivos) y los propósitos formativos. Se reflexiona la práctica pedagógica investigativa desde enfoque hermenéutico como una alternativa para mejorar el ejercicio de la docencia.

Referente a la educación rural se encontraron los siguientes antecedentes:

El artículo titulado "Problemas y retos de la educación rural en Colombia" realizado por (Arias, 2017) de la Universidad Nacional de Colombia, muestra el poco acercamiento de los saberes culturales de las comunidades con la actual educación rural en el país, por su currículo general y deja por fuera los aportes de pobladores de su territorio, lo que hace necesario su reconocimiento a través de los Proyectos educativos rurales (PER).

El artículo titulado "Mejorando el acceso y la calidad de la educación para el sector rural pobre. El caso de la Escuela Nueva en Colombia", realizado por Colbert (1999), describe la problemática de la calidad de la educación rural, muestra como la Escuela Nueva, favorece la atención de los estudiantes para la educación primaria con un docente, por su innovación con la teoría activa del aprendizaje cooperativo y lleva a transformar la práctica pedagógica.

El documento final para un diplomado en gestión educativa territorial en la universidad Pontificia Javeriana, titulado "el sector rural en Colombia y su crisis actual" realizado por Pérez \& Pérez (2002), inicia describiendo la población que habita la zona rural, examina varios factores que desfavorecen el sector rural en Colombia, como el desplazamiento forzado, la violencia, el desempleo y la pobreza, a su vez, presenta un análisis de la tenencia de la tierra, su concentración en propiedad de narcotraficantes y el fenómeno de desplazamiento forzado por la violencia a causa del conflicto armado. Respecto a la educación se presentan porcentajes que demuestran las desventajas que tienen los niños y jóvenes campesinos con respecto al acceso a la educación.

El artículo titulado "la educación rural en Colombia: experiencias y perspectivas" elaborado por Carrero \& González (2016) aborda e análisis histórico de la educción rural en Colombia desde la década de 1950, los modelos educativos que han prevalecido con lineamientos del Ministerio de Educación Nacional como son Escuela Nueva, Aceleración del Aprendizaje, Posprimaria, Telesecundaria, Programa de Educación Continuada Cafam y abre una discusión sobre la educación en tecnología y su favorecimiento para la educación rural y presenta experiencias al respecto en tres 
sedes de una institución educativa de la ciudad de Bolívar.

El artículo "estudio sobre la educación para la población rural en Colombia” presentado por Mauricio Perfetti del Corral (2003), presenta un estado del arte en contribución al proyecto (educación para la población rural) promovido por la Organización de las Naciones Unidas, en esfuerzo colaborativo para la atención de las necesidades de las comunidades rurales que son afectadas por factores socioeconómicos, el desplazamiento forzado por conflictos, la intimidación por grupos al margen de la ley, el analfabetismo y deserción escolar. Se deriva de una investigación que por medio de un enfoque de investigación etnográfico se hace visible las dificultades de la institución educativa para asimilar las nuevas lógicas sociales y favorecer la inclusión de los niños afectados por la violencia política y el desplazamiento, los autores sugieren que comprender los significados que se construyen en la escuela y la necesidad de promover un ambiente escolar que favorezca las relaciones interpersonales con prácticas favorables a la convivencia, el reconocimiento del contexto histórico y social de la escuela como parte de la comunidad y una política pública frente al desplazamiento forzado que reconozca la realidad de las necesidades institucionales y locales.

\section{MARCO HISTÓRICO DE LA EDUCACIÓN RURAL}

El sistema educativo colombiano ha pasado por diversos modelos de escuelas que corresponden a las reformas educativas del siglo XX y principios del siglo XXI que han impactado la educación rural. Aline Helg (1987) su investigación histórica explica la evolución de las tasas de alfabetización, la escolaridad y las funciones de la escuela urbana y rural, en interacción con los maestros y los diferentes tipos de programas de enseñanza que se inculcaban, de acuerdo al precepto oficial, incluyendo la evaluación por la comunidad tanto para el estudiante como para el docente en los diferentes departamentos de Colombia durante la primera mitad del siglo $X X$. Destaca que la educación rural se guiaba por un programa de enseñanza muy similar a la educación urbana, basándose en una didáctica memorística, apoyada en el castigo físico y psicológico. Si bien esto ocurría en la educación oficial, poco se diferenciaba de la educación privada, quienes dirigían este sector, eran en un alto porcentaje órdenes religiosas católicas y particulares, muchos de sus colegios funcionaban en los edificios de la nación o del departamento, pero poco se avanzó en el derecho a la educación.

En la segunda mitad del siglo XX la educación rural ha desarrollado al interior del campo educativo una dinámica en sus prácticas pedagógicas que han mostrado tener profundas influencias e implicaciones para el desarrollo del medio escolar rural. Tal hecho lo muestra ParraSandoval,(1986) en sus estudios donde describe los procesos que han llevado a considerar la escuela como una entidad inacabada e inconclusa en la región rural, por las tensiones entre un modelo de educación que promueve el fortalecimiento de las condiciones y formas de vida del medio rural, ante otra tendencia que trata de imponer un tipo de visión mercantil de la cultura mediante los contenidos que se imparten, chocando de esta forma con la visión del campesino, el indígena y afrodescendiente quien ve la educación como un espacio exógeno que intenta amoldar su forma de vivir y pensar, sin tener en cuenta las condiciones de sus contextos culturales, constituyéndose de esta forma, más en un factor contradictorio, que de avance en la reconstrucción de sus identidades. 
El proceso de modernización colombiano implementado en las dinámicas sociales, políticas y económicas que impactan las transformaciones culturales de este país, han conllevando a la constitución de una escuela rural para la expansión en términos de MartínezBoom, Noguera y Castro (1994), problemática que se manifiesta con mayor fuerza en el contexto cultural bajo la lógica de una práctica pedagógica homogenizante que termina alejando a los niño(a)s campesinos, indígenas y afros de la cultura rural y sus identidades.

Lo preocupante en lo avanzado del siglo XXI es la continuidad de un sistema educativo rural que se deteriora por los diversos problemas sociales de desplazamiento, violencia y pobreza que llevan a la pérdida de capacidad de la educación rural para retener y promover estudiantes como dice la ministra de educación María Victoria Angulo, quien reconoció que la deserción en Educación Media es muy alta y que se agudiza en zonas rurales, los jóvenes terminan estudiando sólo hasta grado noveno (Hernández, 2019a). El Centro de Estudios sobre Desarrollo Económico (CEDE) de la Universidad de los Andes y el Departamento de Diseño de la misma universidad, realizaron una investigación con información detallada sobre el cumplimiento del objetivo de educación de calidad en Colombia, en este estudio se identificaron las causas de deserción en el sector rural: el $34 \%$ de los jóvenes que dejan el colegio lo hacen por problemas económicos; el $28 \%$ por la distancia entre el colegio y la casa, y el $27 \%$ por problemas académicos (Hernández, 2019b).
Igualmente, en el Valle del Cauca esto se ve reflejado en que:

Por cada 100 estudiantes que se matriculan en primero de primaria en las zonas rurales, sólo 35 terminan este ciclo y un poco menos de la mitad (16 estudiantes) pasan a secundaria; de éstos, 8 completan el noveno grado y sólo 7 culminan el ciclo completo de educación básica o media (7\%). Adicionalmente, se encuentran problemas relacionados con: el aprendizaje no significativo, descontextualización de la oferta educativa rural en relación con los cambios científicos y tecnológicos, por lo que se hace necesario involucrar la población rural en la definición de currículos pertinentes. (Gobernación del Valle del Cauca \& Secretaría de Educación Departamental, 2014, p.36).

Son evidentes los grandes problemas que se manifiestan con respecto a los estudiantes en la escuela rural, producto de una serie de reformas educativas que se han implementado en los últimos gobiernos del siglo XXI. En este sentido el Estado Colombiano ha hecho prevalecer la lógica del mercado en el diseño de la política educativa, atribuyéndole al crecimiento económico la cualidad de poder configurar todo, incluido la cultura, lo que induce a implementar programas escolares que convierte al otro (estudiante, directivo y docente) en objeto, alejándolos de la subjetividad sociocultural propia del proceso formativo intercultural. 


\section{ANÁLISIS DE RESULTADOS}

El estudio de la formación en competencias ciudadanas en la educación rural de los municipios objeto de este estudio, se realizó teniendo en cuenta referentes teóricos, específicamente según la clasificación de las competencias ciudadanas MEN (2006) con los siguientes ejes temáticos: 1) convivencia y paz, 2) participación y responsabilidad democrática, 3) pluralidad, identidad y valoración de las diferencias. A continuación, se describen de acuerdo a las respuestas obtenidas.

\section{FORMACIÓN DE COMPETENCIAS} CIUDADANAS EN CONVIVENCIA Y PAZ EN LOS MUNICIPIOS DE TULUÁ Y BUGA EN LOS GRADOS $5^{\circ}, 9^{\circ} \mathrm{Y} 11^{\circ}$.

El análisis de la frecuencia del eje temático convivencia y paz en grado quinto como se describe en la tabla N.3, corresponde al $64,4 \%$ de los estudiantes en de la zona rural del municipio de Tuluá, coincidieron que es la competencia que más se desarrolla en su institución educativa, mientras que en el municipio de Buga solo la consideraron en un $44.4 \%$. Al comparar con la respuesta de los estudiantes de grado noveno y once solo lo manifestaron en un
$49,5 \%$, mientras en el municipio de Buga fue de $12,5 \%$. Notándose una diferencia marcada de su apreciación para el desarrollo del aprendizaje de ejercer la convivencia y la paz como un logro escolar en la construcción de la ciudadanía a medida que avanzan en su nivel educativo, lo que hace necesario para ambos entes territoriales no perder de perspectiva la orientación del MEN de entender las competencias ciudadanas como "el conjunto de conocimientos y habilidades cognitivas, emocionales y comunicativas que, articulados entre sí, hacen posible que el ciudadano actúe de manera constructiva en la sociedad democrática" (2004, p. 8). Lo anterior significa ofrecer la continuidad en la formación ciudadana en los estudiantes de primaria y secundaria necesaria para lograr una convivencia dada desde la educación como posibilitadora de la paz. 
Tabla 3 Comparación de la competencia convivencia y paz en los grados $5^{\circ}, 9^{\circ}$ y $11^{\circ}$ de 2019 de las instituciones rurales de Tuluá y Buga.

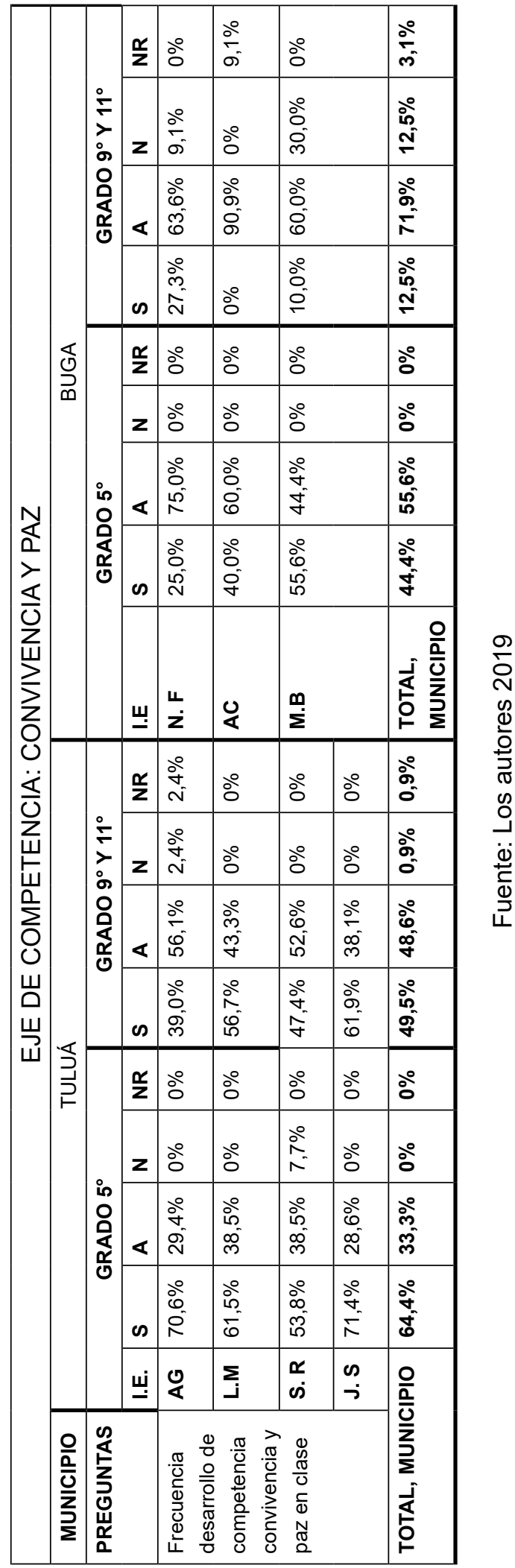

FORMACIÓN DE COMPETENCIAS CIUDADANAS EN PARTICIPACIÓN Y RESPONSABILIDAD DEMOCRÁTICA EN LOS DE TULUÁ Y BUGA EN LOS GRADOS $5^{\circ}, 9^{\circ} \mathrm{Y} 11^{\circ}$.

En la tabla 4, se observa que el $49,1 \%$ de los estudiantes de grado $5^{\circ}$ en la zona rural de Tuluá creen en el logro de la competencia participación y responsabilidad democrática y en un menor porcentaje en Buga con un 38,9\% Al comparar con la respuesta en ambos municipios de los grados noveno y once se nota que los estudiantes de secundaria de Tuluá consideran fundamental la participación de la ciudadanía en un $43,2 \%$, mientras en el municipio de Buga solo en un $34,4 \%$. Notándose esta tendencia de su baja apreciación por los estudiantes de este ente territorial en dicho eje temático de la responsabilidad democrática. En este sentido Urzúa y De Puelles (1996), proponen que la manera de llegar a una ciudadanía democrática es fundamental la formación en los valores universales de la democracia participativa "Ios valores relativos a la tolerancia, la autonomía y los derechos humanos; los relacionados con la práctica de la deliberación conjunta, la solución pacífica de los conflictos y la responsabilidad por las decisiones; los relacionados con la solidaridad"( pp. 10-11).

Lo anterior significa la necesidad de ampliar en los currículos la promoción de la historia de Colombia en estas Instituciones educativas para que los estudiantes consoliden en su práctica ciudadana la participación en su comunidad para que entiendan la importancia de desarrollar las capacidades para el consenso y el diálogo en la construcción de soluciones en su localidad y nación. 
Tabla 4 Comparación de la competencia participación y responsabilidad democrática según los grados $5^{\circ}, 9^{\circ}$ y $11^{\circ}$ de 2019 de las instituciones rurales de Tuluá y Buga.

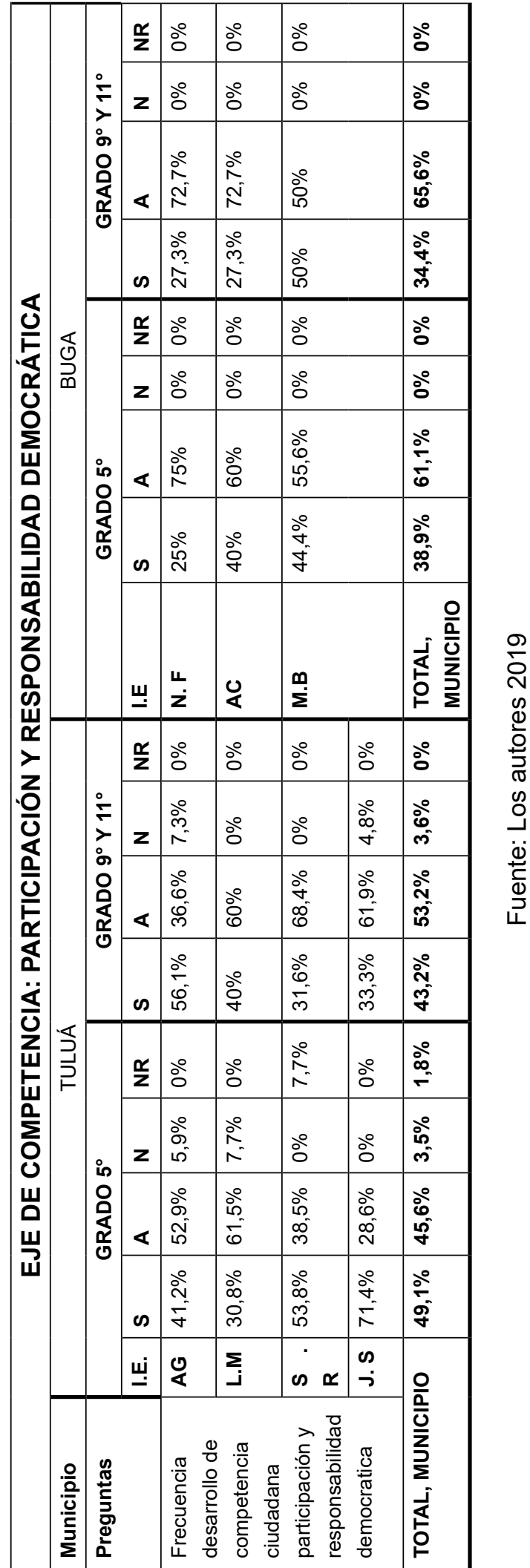

FORMACIÓN DE COMPETENCIAS CIUDADANAS EN PLURALIDAD, IDENTIDAD Y VALORACIÓN DE LAS DIFERENCIAS EN LOS MUNICIPIOS DE TULUÁ Y BUGA EN LOS GRADOS $5^{\circ}, 9^{\circ} \mathrm{Y} 11^{\circ}$.

El análisis de la frecuencia de desarrollo del eje temático pluralidad, identidad y valoración de las diferencias en grado quinto como se describe en la tabla 5, corresponde al $54,4 \%$ de los estudiantes de grado quinto en de la zona rural del municipio de Tuluá, coincidieron que es una de las competencias que se trabaja en su Institución educativa, pero con una mayor tendencia en el municipio de Buga en los estudiantes del grado mencionado en un $77,8 \%$. Al compararla con la respuesta de los estudiantes de grado noveno y once en Tuluá se conserva la tendencia con un 55\%, a diferencia del municipio de Buga sólo el $31,2 \%$ de los estudiantes de grado noveno y once, reconocen el trabajo de este eje temático. 
Tabla 5 Comparación de la competencia pluralidad, identidad y valoración de las diferencias según los grados $5^{\circ}, 9^{\circ}$ y $11^{\circ}$ de 2019 de las instituciones rurales de Tuluá y Buga.

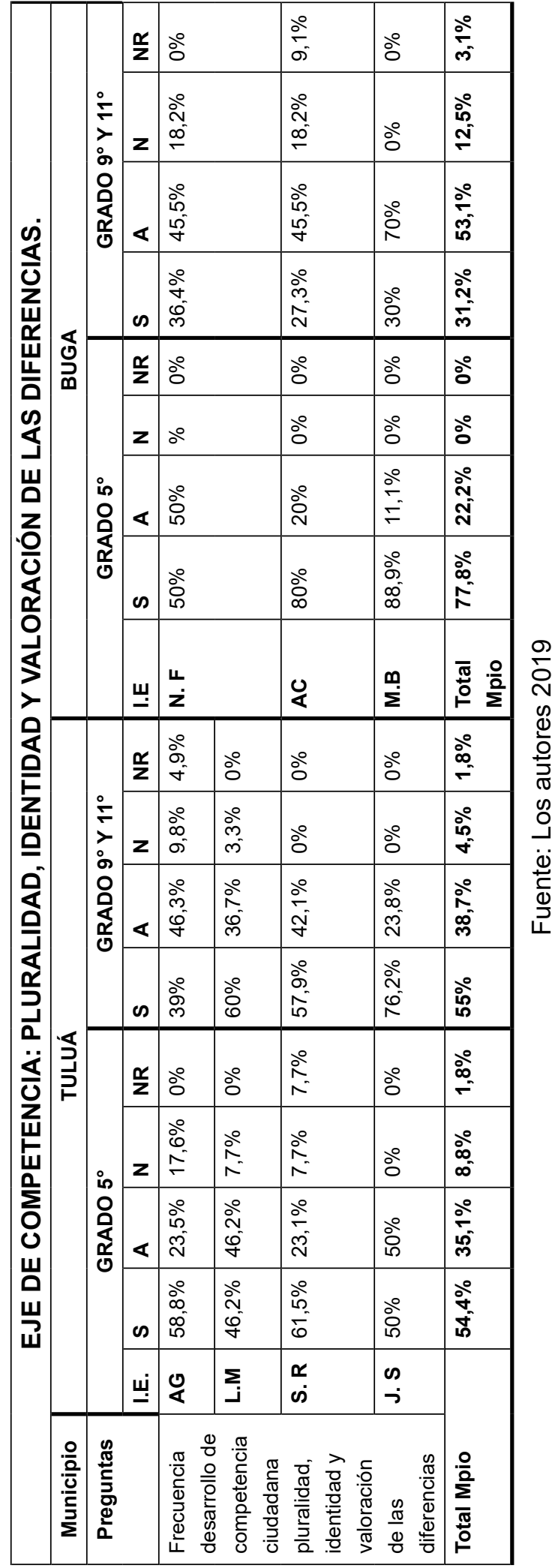

Con respecto al grado de identidad de los estudiantes como campesinos, se pudo evidenciar en la tabla 6 que los estudiantes encuestados tanto del municipio de las instituciones educativas rurales del municipio de Buga (55,6\% del grado quinto) como los de Tuluá $(50,9 \%)$ respondieron afirmativamente, pero al comparar la respuesta con los estudiantes de noveno y once es notorio la no aceptación como campesinos en Buga de un 56,2\% y de Tuluá en un $55 \%$. 
Tabla 6. Comparación de la competencia pluralidad, identidad y valoración de las diferencias en su condición de campesino según los grados $5^{\circ}, 9^{\circ}$ y $11^{\circ}$ de 2019 de las instituciones rurales de Tuluá y Buga.

\begin{tabular}{|c|c|c|c|c|c|c|}
\hline & $\frac{\infty}{2} \frac{c}{z}$ & ঃ̊ & & ठ̊ & ठ̊ & ঃั \\
\hline & 올 & 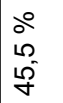 & & $\begin{array}{l}\circ \\
\infty \\
\infty \\
\infty \\
\infty\end{array}$ & 品 & $\begin{array}{l}\text { సे̀ } \\
\text { î̀ }\end{array}$ \\
\hline : & 'क & \begin{tabular}{|l|}
$n$ \\
0 \\
0 \\
10 \\
\end{tabular} & & \begin{tabular}{|l|}
$N$ \\
$\infty$ \\
$\sim$ \\
-
\end{tabular} & $\begin{array}{l}\circ \\
\stackrel{\circ}{\circ}\end{array}$ & 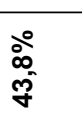 \\
\hline s & $\bar{\infty}_{z} \frac{\alpha}{z}$ & ○̊ & & ঃ̊ & ○े & ঃ̊ \\
\hline త్ & 을 & ஃ̊ & & \begin{tabular}{|l}
$\circ$ \\
0 \\
0 \\
$\infty$
\end{tabular} & 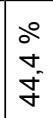 & $\begin{array}{l}\stackrel{\circ}{\grave{j}} \\
\dot{f}\end{array}$ \\
\hline$\infty$ & 's & $\begin{array}{l}0 \\
0 \\
0 \\
8 \\
0\end{array}$ & & $\begin{array}{l}\circ \\
0 \\
0 \\
\text { N }\end{array}$ & $\begin{array}{l}0 \\
0 \\
0 \\
0 \\
0 \\
0\end{array}$ & 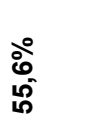 \\
\hline & بِ & $\begin{array}{l}\ddot{L} \\
\dot{z}\end{array}$ & & ¿ & $\stackrel{m}{\Sigma}$ & $\begin{array}{l}\text { 巨ٓ } \\
\stackrel{\circ}{\circ}\end{array}$ \\
\hline & o & $\begin{array}{l}\circ \\
0 \\
0 \\
0 \\
\infty\end{array}$ & $\begin{array}{l}\circ \\
\circ \\
\circ\end{array}$ & $\begin{array}{l}\circ \\
0 \\
0 \\
0 \\
\text { N }\end{array}$ & $\begin{array}{l}0 \\
0 \\
0 \\
0 \\
0\end{array}$ & 今ั \\
\hline $\mid$ & क & 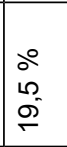 & $\stackrel{\circ}{2}$ & $\begin{array}{l}\stackrel{0}{2} \\
\hat{N} \\
\end{array}$ & $\begin{array}{l}\circ \\
0 \\
m \\
m \\
m\end{array}$ & ๖े \\
\hline & $\frac{\infty}{z} \frac{\alpha}{z}$ & ஃ̊ & ○̊ & ○े & $\begin{array}{l}\circ \\
\therefore \\
i\end{array}$ & ڤ̊ \\
\hline$\varangle$ & 을 & $\begin{array}{l}\circ \\
0 \\
0 \\
0\end{array}$ & $\begin{array}{l}\stackrel{0}{0} \\
i n \\
\infty \\
\infty\end{array}$ & ঃ̊ & 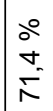 & 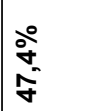 \\
\hline $\overrightarrow{2}$ & "ळ & $\begin{array}{l}\stackrel{\circ}{\dot{0}} \\
\text { ळे }\end{array}$ & $\begin{array}{l}0 \\
10 \\
5 \\
6\end{array}$ & $\begin{array}{l}\circ \\
0 \\
0 \\
8 \\
0\end{array}$ & $\begin{array}{l}\stackrel{0}{\circ} \\
\dot{\sigma} \\
i \\
i\end{array}$ & 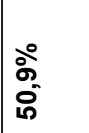 \\
\hline c & بِّ & Q & $\sum_{j}$ & $\begin{array}{l}\alpha \\
\omega\end{array}$ & os & \\
\hline $\begin{array}{l}\frac{0}{0} \\
\frac{0}{Z} \\
\frac{D}{\Sigma}\end{array}$ & 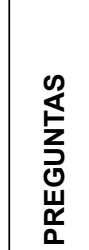 & 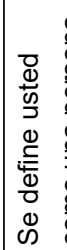 & 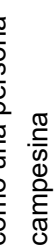 & & & 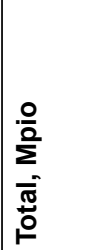 \\
\hline
\end{tabular}

A manera de tendencias al comparar la formación en competencias ciudadanas en la educación rural de los municipios objeto de este estudio, la de mayor reconocimiento es dada por los estudiantes rurales de Tuluá en el grado quinto, noveno y once correspondiente a convivencia y paz. Siguiéndole el eje temático pluralidad, identidad y valoración de las diferencias para la población escolar, mientras la de menor impacto en los estudiantes de la zona rural seria la competencia participación y responsabilidad democrática.

En el caso de Buga la tendencia en formación en competencias ciudadanas en la educación rural la de mayor reconocimiento por los estudiantes del grado quinto, noveno y once corresponde al eje temático pluralidad, identidad y valoración de las diferencias. Siguiéndole el eje temático participación y responsabilidad democrática para la población escolar, mientras la de menor impacto fue el eje temático convivencia y paz.

Además, tomando como referencia los resultados de las entrevistas a los estudiantes de grado quinto, noveno y once con respecto a la formación en competencias ciudadanas, se lograron los siguientes hallazgos y categorías emergentes:

\section{- $\quad$ LA CONVIVENCIA UN APRENDIZAJE CLAVE PARA LA VIDA}

Los estudiantes coinciden con los docentes frente a las competencias ciudadanas en la categoría Convivencia y paz, es relevante la valoraron en sus respuestas como un aprendizaje clave para la vida. Por ejemplo, para los estudiantes de grado $11^{\circ}$, en la clase han dialogado sobre la importancia "porque las competencias ciudadanas nos permiten a nosotros los ciudadanos tener una convivencia pacífica, para poder vivir con los otros y actuar de manera constructiva en la sociedad" I.E.N.S.F.(E1). También, se encontró que se considera importante que los docentes propicien 
este aprendizaje articulado a los temas que van desarrollando en las clases: "Es muy fundamental, desde pequeños ellos nos explican y nos dan a entender todo lo que necesitábamos para convivir entre tanta gente" I.E.A.C.(E4).

Así mismo, para estudiantes de $9^{\circ}$ grado, por ejemplo: "Las competencias ciudadanas, nos ayudan a actuar de mejor manera como humanos, esto nos ayuda en nuestra vida para saber convivir con las demás personas" I.E.A.C.(E5). Por su parte, para algunos estudiantes de $5^{\circ}$ grado, este aprendizaje es importante porque "nos enseña que debemos tener una convivencia adecuada" I.E.A.C.(E6), "Para respetarnos, para que uno pueda vivir en convivencia" I.E.M.(E9)

\section{- $\quad$ LAS COMPETENCIAS CIUDADANAS SON UN APRENDIZAJE PARA LA CONSTRUCCIÓN SOCIAL}

Según lo manifestado por estudiantes en las entrevistas, las competencias ciudadanas se consideran de utilidad para hacer parte de una sociedad, algunos comentarios al respecto: respuestas de estudiantes de $11^{\circ}$ "permiten actuar de manera constructiva en la sociedad." I.E.N.S.F.(E1). También sirven para "Hacer posible que las personas participen en la construcción de una sociedad democrática y pacífica". I.E.A.C.(E4). En los estudiantes de $9^{\circ}$ se encontó "Es necesarias, porque estamos en un proceso de formación donde todo lo que se aprende se debe aplicar en la sociedad y para la sociedad" I.E.N.S.F.(E2), "lo aprendido lo socializamos con la comunidad" I.E.M.(E7).

En la educación por competencias es fundamental la perspectiva integradora de los procesos curriculares para lograr la formación integral del ser humano, lo que requiere tener claridad que trabajar de manera integrada las competencias, es mucho más que trabajar cada una de sus dimensiones de manera separada. Como lo explica De Zubiría (2013) "pensar la educación desde la integralidad implica reivindicar la interdependencia y el carácter holístico de las competencias como principio fundamental" (p.32), teniendo en cuenta que "Es obligación enseñar a pensar mejor, a amar mejor y a actuar mejor" (p. 30). Lo que suscita plantearse la construcción curricular como un proceso social y cultural.

\section{CONCLUSIONES}

La educación en competencias ciudadanas en las Instituciones educativas rurales en los municipios de Guadalajara de Buga y Tuluá, presentan diferentes procesos de formación en la práctica pedagógica intercultural. En las experiencias vividas por los estudiantes con su comunidad educativa, desde el nivel educativo de la primaria donde reconoce la manera de relacionarse entre las personas y con su cultura, se identificó su conocimiento y apropiación de algunas habilidades sociales sobre Convivencia y Paz en los estudiantes de la zona rural de Tuluá y Buga, presentándose fortalecimiento de esta competencia en los estudiantes de noveno y once del municipio de Tuluá, mientras en el caso de Buga, se evidenció menor valoración de esta competencia para su vida cotidiana como ciudadanos. Lo anterior significa la necesidad de revisar la coherencia del modelo educativo por competencias en su continuidad entre la primaria y la secundaria para que no pierda el peso en la formación ciudadana como posibilitadora de la paz, reconociendo las particularidades propias de cada institución educativa y del ente territorial.

Si bien, la Constitución de 1991 nos aporta las bases para la construcción de una democracia participativa en Colombia, es necesario recrear estrategias pedagógicas y didácticas que no se agoten en lo procedimental y se avance en procesos de pensamiento y en el desarrollo de procesos socio afectivos que les permita hacer lectura del contexto sociocultural y político. De estar en capacidad activa de resolver las necesidades propias y de su entorno, en este 
sentido los estudiantes de grado $5^{\circ}$ en la zona rural de Tuluá y Buga creen en la participación y responsabilidad democrática, pero va decreciendo en ellos esta percepción en ambos municipios, a medida que avanzan en los grados noveno y once. Se va perdiendo el interés por la democracia participativa y se da relevancia a la representativa como símbolo de delegar a otro la responsabilidad en la toma de decisiones de lo que atañe a todos e incluye la diversidad cultural, además se identificó el desengaño ante la política tradicional. Es necesario seguir fortaleciendo el desarrollo de las competencias ciudadanas en la perspectiva de un pensamiento participativo, deliberativo, la búsqueda de soluciones con el voto programático y la construcción de una responsabilidad ciudadana en la toma de las decisiones para ampliar la noción de democracia.

Una práctica pedagógica intercultural puede aportar al desarrollo del eje temático pluralidad, identidad y valoración de las diferencias, se observó como en los estudiantes de la educación de grado quinto de los municipios estudiados la mayoría coincidieron en dar importancia a este eje, con una mayor tendencia en el municipio de Buga, pero se fragmenta en los grados noveno y once frente al desarrollo del pluralismo político, lo que hace necesario una reconceptualización del modelo educativo por competencias desde los aportes de la educación intercultural a partir del enfoque sociocultural de la pedagogía, que permita entender el desarrollo rural y el bienestar de la comunidad campesina, afro e indígena sin caer en el sin sentido de la modernización a ultranza del desarrollismo económico y avanzar en una interpretación desde la expresión del reconocimiento de la existencia de diferentes comunidades culturales, para poder llegar a la autocomprensión como sujetos culturales en permanente relación y reconstrucción de sentidos de un ciudadano activo.
La identidad cultural como expresión de la memoria colectiva, registra las representaciones de un grupo de personas (Candau, 2008), las cuáles poseen una memoria compartida de la vida social, familiar, laboral y que a la vez dan cuenta de las dinámicas socioculturales, económicas, políticas que han incidido en sus experiencias de vida. Además, debe ser entendida como proceso intersubjetivo de interacción y comunicación con significaciones históricas que sitúan en tiempo y espacio y que implican la otredad, el reconocimiento del otro como ser autentico y diferente "el reconocimiento de sí mismo, el reconocimiento hacia otros y el reconocimiento de otros hacia nosotros" (Marcús, 2011, p.108). La identidad como proceso cultural se construye y reconstruye en la relación consigo mismo y con el otro "La conciencia del hombre despierta envuelta en la conciencia ajena" (Bajtín, 1982, p.360). № es el reconocer que existen diversidad de culturas lo que posibilita la capacidad de comprender y ponernos en el lugar del otro, lo que permite es acceder a los contenidos simbólicos que nos unen y permiten establecer comunicación "me permite acceder a lo que me vincula a él, a esos objetos en los que podemos reconocernos ambos" (Merieu, 1998, p.134), por lo tanto, es imperativa una educación intercultural que enfatice en la formación del reconocimiento del otro. En un proceso educativo intercultural se puede aportar a la construcción de identidad reconociendo los relatos autobiográficos entrecruzados, que hilan el tejido de una biografía colectiva de las comunidades rurales de estos municipios.

La vida en el contexto rural de estos municipios está acompañada de tensiones como es la distribución del territorio entre pequeños propietarios que viven y trabajan en el campo, grandes hacendados y dueños de predios que utilizan para el esparcimiento en el campo. De acuerdo a las entrevistas se mencionan aspectos positivos, resaltando la tranquilidad que ofrece el paisaje rural, es decir el contacto 
con la naturaleza, la vida sencilla sin el afán del consumismo, la solidaridad entre los vecinos, el reconocimiento a quien tiene experiencias y saberes para compartir con las nuevas generaciones, las costumbres y tradiciones que se conservan, entre otros aspectos. Sin embargo, existen problemáticas sociales, políticas y económicas que enrarecen la tranquilidad de las personas de diverso origen cultural que habitan el campo, que se ven afectadas por situaciones de desempleo, violencia, inseguridad, desplazamiento, entre otros. Algunos logran encontrar alternativas de sobrevivencia, otros menos afortunados hacen parte de la lista de víctimas del conflicto, de la población vulnerable en situación de pobreza o de miseria en calidad de desplazados.

En la búsqueda de equilibrio entre la otredad y la alteridad se puede avanzar en procesos educativos interculturales, donde es categórico entender al otro como diferente, tratar de ponerse en su lugar y entender su sentir y su razonar. Es en el reconocimiento de la alteridad que se puede entender el sentido diverso y dialógico de la identidad cultural, "una cultura puede extender sus horizontes y enriquecer sus fuentes intelectuales y espirituales al aprender de visiones alternativas en epistemología, ética, estética y perspectivas de vida, cuando es críticamente consciente de sus propias fortalezas y debilidades" (UNESCO, 2013, p. 4 ), es en el encuentro con la propia cultura que podemos tener la sensibilidad para reconocer, respetar y valorar la existencia de otras culturas y sólo entonces, se puede plantear una educación intercultural que además, llegue a trascender con elementos culturales glocales. En el caso de la educación rural es necesario reforzar la identidad de la cultura campesina, para garantizar el desarrollo de capacidades que hagan posible establecer dialogo con otras culturas sin exponer a los estudiantes al desencanto de su propia cultura sin conciencia de identidad.
Ante la realidad que caracteriza la vida en el campo es imperativo plantear una educación que propicie una formación que potencie la resiliencia; porque es fundamental desarrollar en los niños y jóvenes campesinos las capacidades (Nussbaum, 2012), para enfrentarse a las adversidades y obstáculos; igualmente para reflexionar la comprensión de las problemáticas y la búsqueda de alternativas de solución, lo que les permita lograr superarse, incluso sacar provecho de las experiencias que acompañan sus historias de vida (Grotberg, 1995). Una educación intercultural que aporte capacidades para ser creativos ante las situaciones difíciles, actuando con valores y principios éticos; es así que, la UNESCO respecto a las competencias interculturales plantea que la creatividad es la que "nos brinda resiliencia para adaptarnos a diferentes ecosistemas y para inventar «modos de vivir juntos»" (UNESCO 2013, p. 22). Sobre todo, para construir un mundo responsable y respetuoso con la educación ciudadana de los niños, los jóvenes y de todas las personas que nos posibilitan la seguridad alimentaria al resto de los pobladores.

\section{REFERENCIAS BIBLIOGRÁFICAS}

Albán, A. (2012). La educación intercultural ante el desplazamiento forzado: ¿Posibilidad o quimera? Contextos, 1(1), 55-66. Colombia: Universidad Santiago de Cali. Consultado en: https://repository.usc.edu.co/bitstream/ handle/20.500.12421/573/16-29-1-SM. pdf? sequence $=1$ \&isAllowed $=y$

Alomía, M. (2015). Una reflexión crítica desde la educación intercultural hacia la pedagógica de la recreación. Lúdica pedagógica, No. 22 (2015 - II) 45 SECCIÓN CENTRAL pp. 45-53. Consultado en: file:///C:/Users/SONY/ Downloads/Una_reflexion_critica_ desde la educacion intercult.pdf 
Arias, J. (2017) "Problemas y retos de la educación rural en Colombia" Educación y Ciudad No33, pp.53-61. Consultado en: https://revistas.idep. edu.co/index.php/educacion-y-ciudad/ article/view/1647/1623

Bajtín, M. (1982). Estética de la creación verbal, México: Siglo XXI Editores.

Burgos, D. \& Cifuentes, J. La práctica pedagógica investigativa: entre saberes, quereres y poderes. Revista Horizontes Pedagógicos 17(2), 118127. Consultado en: file:///C:/Users/ SONY/Downloads/Dialnet-LaPracticaP edagogicalnvestigativa-5455073.pdf

Candau, J. (2008). Memoria e identidad. Buenos Aires: Ediciones Del Sol.

Carrero, M. Arango y González, M. (2016). La educación rural en Colombia: experiencias y perspectivas. Praxis Pedagógica. No.19 julio-diciembre 2016 ISSN 0121-1494. pp: 79-89. Consultado en: file:///C:/Users/SONY/Downloads/ La_educacion_rural_en_Colombia_ experiencias_y_pers.pdf

Colbert, V. (1999) "Mejorando el acceso y la calidad de la educación para el sector rural pobre. El caso de la Escuela Nueva en Colombia". Revista Iberoamericana $n^{\circ}$ 20, pp.107-135. Consultado en: https://rieoei.org/historico/documentos/ rie20a04.PDF

Departamento Administrativo Nacional de Estadística DANE. (2019). Grupos étnicos información técnica. Colombia. Consultado en: https://www.dane.gov. co/index.php/estadisticas-por-tema/ demografia-y-poblacion/grupos-etnicos/ informacion-tecnica
Departamento Nacional de Planeación. (1998). Cambios para construir la paz. 1998 - 2002. Santa Fé de Bogotá: Tercer Mundo Editores.

Gastón, R. (2016). El ámbito científico de la formación política: elementos conceptuales y líneas de investigación. Educación y educadores. $\mathrm{n}^{\circ} 2$ (19), 177-306 p., mayo- agosto. Colombia: Universidad de la Sabana.

Gobernación del Valle del Cauca \& Secretaría de Educación Departamental. (2014). La cobertura educativa en el ente territorial Valle del Cauca. Razones y algunas explicaciones de su variación. Consultado el 14 de marzo de 2017, disponible en: file:///C:/Users/mealvarez/ Downloads/La Cobertura Educativa En El Ente Territorial Valle Del Cauca.pdf

Gobernación del Valle del Cauca \& Secretaría de Educación Departamental. (2012). Plan sectorial de educación 2012 - 2015. Consultado el 14 de marzo de 2017, disponible en: $\quad$ http://vallecaucanadeaguas. gov.co/documentos/04PLAN\%20 SECTORIAL\%20DE\%20EDUCACION. pdf

Helg, A. (2001). La educación en Colombia 19181957. Una historia social, económica y política. Bogotá: Universidad Pedagógica Nacional.

Guerrero, J. (2014). La interculturalidad usos y abusos, en: Pedagogías otras: diversidad e inclusión. Educación y Ciudad No 26, enero - junio de 2014 ISSN 0123-0425 - pp. 15-28 
Guido, S. (2010). Interculturalidad y política educativa en Colombia. Interculturalidad y política educativa en Colombia. Revista Internacional Magisterio, V. 46, p.32-37.

Mejía, O. (2011). La cultura mafiosa en Colombia y su impacto en la cultura jurídico- política. Pensamiento jurídico $n^{\circ} 30$, enero- abril, Bogotá, pp. $15-62$. Consultado en: https://doctrina.vlex. com.co/vid/mafiosa-colombia-mafiapolitical-351100258

Merieu, P. (1998). Frankenstein educador. Barcelona: Editorial Laertes S.A.

Ministerio de Educación Nacional (2010). Manual de implementación Escuela Nueva. Generalidades y orientaciones pedagógicas para transición, primer grado. Tomo I. Bogotá: MEN.

Ministerio de Educación Nacional (2004). Estándares básicos de competencias ciudadanas. Bogotá: MEN.

Ministerio de Educación Nacional. (2001). Serie lineamentos curriculares Cátedra estudios afrocolombianos. Consultado en: https://www.mineducacion.gov. co/1759/articles-339975_recurso_2.pdf

Nussbaum, M. y Sen, A. (1996) La calidad de vida. México: Fondo de Cultura Económica.

Nussbaum, M. (2011). Sin fines de lucro, Porque la democracia necesita de las humanidades. Bogotá: Katz editores, $\mathrm{p}$, 20.

Nussbaum, M. (2012). Crear capacidades. Propuesta para el desarrollo humano. Barcelona: Paidos.
Parra-Sandoval, R. (1986). Clases sociales y educación en el desarrollo de Colombia. En: U.N. Revista de la Dirección

Pérez, E. \& Pérez, M. (2002). El sector rural en Colombia y su crisis actual. Cuadernos de Desarrollo Rural (48), pp. 35-58. Consultado en: file:///C:/Users/SONY/ Downloads/1993-Texto\%20del\%20art\% C3\%ADculo-6779-1-10-20120228.pdf

Pinzón, M. (2010). Anotaciones para una pedagogía intercultural en contextos urbanos. Tema: Diversidad cultural y educación. Revista Internacional Magisterio N 46 septiembre - octubre, Bogotá. Consultado en: https://issuu. com/revistamagisterio/docs/revista46/5

Riveros, A. (2010) Interculturalidad, practicas pedagógicas de maestros y maestras y construcción del otro. Revista GrafíaCuaderno de trabajo de los profesores de la Facultad de Ciencias Humanas. Universidad Autónoma de Colombia, [S.I.], n. 7, p. 131-152, may 2015. ISSN 2500-607X. Disponible en: <http:// revistas.fuac.edu.co/index.php/grafia/ article/view/213>. doi:http://dx.doi. org/10.26564/16926250.213.

Sánchez, I. (2011). Modelos y enfoques de educación intercultural. Revista Praxis No. 72011 ISSN: 1657-4915 Págs. 30 - 41 Santa Marta, Colombia. Consultado en: file:///C:/Users/SONY/Downloads/ Dialnet-EnfoquesYModelosDeEducacio nlntercultural-5907175.pdf

Unesco. (2013). Intercultural competences. Conceptual and operational framework. Unesco. París. Recuperado de https:// en.unesco.org/interculturaldialogue/ resources/132 
Urzúa, R \& De Puelles, M. (1996). Documento de consulta presentado a la IV Conferencia Iberoamericana, utilizado como base para la elaboración de la "Declaración de Concepción" [en línea]. Disponible en: http://www.oei.es/oeivirt/rie12a05. htm. [Consultado 10 de agosto del 2020].

Vasco, E. (1997). Maestros, alumnos y saberes. Investigación y docencia en el aula. Colombia: Cooperativa Editorial Magisterio.

Zuluaga, O., Echeverri A., Martínez, A., Quiceno, H., Sáenz J. \& Álvarez A. (2011) pedagogía y epistemología. Bogotá: Editorial Magisterio. Consultado en: $\quad$ https://mefistocastellano. files.wordpress.com/2015/09/ zuluaga-y-otros-pedagogc3ada-yepistemologc3ada.pdf 